\title{
Análisis de la adaptación de la docencia virtual universitaria durante la COVID-19
}

\section{Analysis of the adaptation of online university teaching during the COVID-19 times}

José Garres-Díaz ${ }^{1}$, Jónatan Herrera-Fernández², Alma L. Albujer-Brotons³, Magdalena Caballero-Campos ${ }^{4}$ \& Tomás Morales de Luna ${ }^{5}$

Fecha de recepción: 20/04/2021; Fecha de revisión: 03/07/2021; Fecha de aceptación: 05/10/2021

Cómo citar este artículo:

Garres-Díaz, J., Herrera-Fernández, J., Albujer-Brotons, A.L., Caballero-Campos, M., \& Morales de Luna, T. (2021). Análisis de la adaptación de la docencia virtual universitaria durante la COVID-19. Revista de Innovación y Buenas Prácticas Docentes, 10(2), pp. 131-157.

\footnotetext{
1 Universidad de Córdoba (España), jgarres@uco.es

2 Universidad de Córdoba (España), jherrera@uco.es

3 Universidad de Córdoba (España), alma.albujer@uco.es

4 Universidad de Córdoba (España), magdalena.caballero@uco.es

5 Universidad de Córdoba (España), tomas.morales@uco.es
} 
Presentamos un estudio que analiza la opinión del estudiantado sobre la adaptación a la docencia online realizada en la Universidad de Córdoba durante la crisis sanitaria de la COVID-19. Lo inesperado de la situación acaecida condujo al uso de distintas y muy variadas estrategias para la virtualización por parte del profesorado. El objetivo de este estudio ha sido analizar distintas cuestiones: la adaptación de la docencia a un formato virtual por parte del profesorado, la valoración de la docencia presencial frente a la virtual, el tipo de docencia recibida (síncrona, asíncrona o una combinación de ambas), al igual que la opinión sobre la misma, y el uso y valoración de las herramientas de videoconferencia y comunicación más utilizadas. Además, hemos realizado un análisis y una comparativa de dichas herramientas de videoconferencia. El estudio se ha realizado a través de la elaboración de una encuesta dirigida al estudiantado. La alta y heterogénea participación obtenida, nos permite obtener una visión muy general de la eficacia y valoración de las distintas aproximaciones, así como algunas conclusiones de interés de cara a la organización futura de la docencia.

Palabras clave: Calidad de la enseñanza, cambio tecnológico, enseñanza superior, tecnologías de la información y de la comunicación

\section{Abstract:}

A study is presented based on the students' opinion, analyzing the adaptation of the online teaching in the University of Córdoba during the COVID-19 health crisis. As consequence of the sudden crisis, different strategies and tools were used by teachers for the virtualization of the university teaching. The main goals of this study are to analyze: how teaching has been adapted to the online framework by teachers, how this online teaching is evaluated by students when compared to classical on-site teaching, which type of teaching has been mostly used (synchronous, asynchronous or both), how these approaches are valued, and which are the uses and assessment of the most popular video-conference programs. Moreover, an analysis and comparison of video-conferencing software will be presented. In order to carry out this study a survey for students has been drawn up. Thanks to the high and heterogeneous students' turnout in this study, it is possible to obtain an overview of the efficiency and evaluation of the different approaches used in online teaching. Some relevant conclusions may be drawn for future similar case scenarios.

Key Words: Higher education, information and communication technologies, teaching quality, technological change 


\section{INTRODUCCIÓN}

La amenaza sanitaria que la COVID-19 ha constituido y sigue constituyendo está generando una ola tecnológica provocada por la imposibilidad de continuar con el método tradicional de trabajo, obligando a empresas y administraciones públicas a rediseñar su estructura y procesos. La universidad no ha escapado a esta revolución digital que se ha impuesto a marchas forzadas.

La situación en la que la universidad española se encontraba antes del cambio de paradigma que hemos experimentado en los últimos meses se ve recogida en los últimos informes de CRUE Universidades Españolas. Las universidades habían comenzado el camino hacia la digitalización, incluyendo como objetivos concretos en el eje enseñanza-aprendizaje el proporcionar soporte y promover la docencia no presencial (UNIVERSITIC 2017, 2017). El uso de plataformas soporte a la docencia era masivo, aunque superficial, por lo que se requería explotar al máximo las capacidades analíticas de las mismas (UNIVERSITIC 2016, 2016), considerándose que el mayor desafío era la falta de habilidades digitales de los docentes (Informe de situación de las tecnologías educativas, 2018). Finalmente se planteaba el reto de cambiar de modelo de universidad adaptándolo a la cuarta revolución industrial, teniendo como punto de referencia que nuestros "clientes", los alumnos, consumen y demandan servicios de manera distinta a como se vienen ofreciendo, y asumiendo que la digitalización de la universidad en el siglo XXI, como la de cualquier otro sector, no es una opción (Grupo de trabajo de directores TI, 2017).

Más allá de los anteriores informes, queremos citar algunos estudios que apuntan en la línea del papel central del alumnado en esta transformación digital. LópezPérez, Pérez-López y Rodríguez-Ariza (2011) analizan la percepción de los estudiantes del b-learning. Gisbert y Esteve (2011) contemplan las competencias digitales de los estudiantes en el entorno de la docencia como clave para evitar una brecha que no es evidente debido a las características de la generación a la que el alumnado pertenece. En Universidad 2020 (2012) se destaca el papel crucial de las TIC en el futuro de una institución prácticamente inamovible desde sus inicios y a la que llega un nuevo perfil de alumnado, los nativos digitales. En 2015 un informe de PwC (Price Waterhouse Coopers), The 2018 digital university (n. d.), analiza las experiencias del estudiante en las universidades británicas que lideran procesos de transformación digital, y Newman y Beetham (2017) establecen indicadores de impacto para la experiencia digital del estudiante en las anteriores universidades.

Estas herramientas digitales se han vuelto imprescindibles a lo largo del curso 2019/2020 a causa de la crisis de la COVID-19, ya que la práctica totalidad de las universidades públicas españolas se vio forzada a virtualizar su docencia. Las TIC pasaron de ser usadas de forma mayoritaria como una herramienta que aportaba un valor añadido, a convertirse repentinamente en un eje transversal del proceso enseñanza-aprendizaje. El inicio de este proceso de virtualización, dada la urgencia de la situación, se realizó en general de una manera poco uniforme, siendo dicho proceso llevado a cabo por cada profesor de forma independiente. 
Nos centramos en este trabajo en el caso de la Universidad de Córdoba (UCO), aunque este estudio puede considerarse extrapolable al conjunto de las universidades públicas españolas. En el momento de inicio de la crisis, la UCO tenía preparada (al menos en términos prácticos) dos herramientas para la virtualización de la docencia: la plataforma Moodle y una aplicación para videoconferencias (integrada en la anterior plataforma), Blackboard Collaborate. Sin embargo, la infraestructura disponible no estaba preparada para la enorme carga de trabajo que suponía una virtualización completa de toda la docencia de la Universidad, siendo necesario limitar el uso de la misma temporalmente.

Esta situación forzó a todo el profesorado de la UCO a tener que virtualizar sus asignaturas a través de métodos distintos, ya fuera llevando a cabo una aproximación asíncrona a la docencia, mediante la subida de documentación a la plataforma Moodle (presentaciones con y sin voz, documentos de apoyo, vídeos en plataformas de distribución como YouTube, etc.), una aproximación síncrona con el uso de videoconferencias (usando plataformas como Zoom, Microsoft Teams, etc.) o mediante una combinación de las mismas.

Nuestro objetivo en este artículo es analizar, mediante una encuesta realizada a estudiantes de la UCO, cómo ha sido la virtualización de la docencia desde el punto de vista del alumnado, así como su satisfacción con dicho proceso, tratando de encontrar qué sistemas son aquellos, de los que han hecho uso, que consideran más adecuados y útiles. Deseamos que estos datos sean de utilidad de cara a la planificación de la virtualización de la enseñanza para los próximos cursos, no solo en la UCO, sino también el resto de instituciones de enseñanza superior.

El artículo está estructurado como sigue: en la sección 2 se detalla el desarrollo de la experiencia de innovación, incluyendo los objetivos de este estudio, expresados en forma de preguntas a las que se pretende dar respuesta. En esta sección se establecerá también una comparativa objetiva entre las distintas herramientas de videoconferencia consideradas. En la sección 3 se presentan los resultados obtenidos, tanto respecto al perfil de los encuestados como las respuestas a las distintas preguntas formuladas, describiendo los resultados obtenidos de la encuesta realizada a los estudiantes. En la sección 4 se presentan algunas conclusiones, y finalmente se incluye un anexo con la encuesta utilizada para este estudio.

\section{DESARROLLO DE LA EXPERIENCIA DE INNOVACIÓN}

En la Universidad de Córdoba (UCO), al igual que en el resto de universidades españolas, la pandemia ocasionada por la COVID-19 obligó a pasar a una modalidad completamente online durante el curso 2019/2020. Debido a la urgencia de la situación, la adaptación hubo de hacerse sobre la marcha, utilizando las herramientas existentes, muchas de ellas desconocidas, según el criterio del profesor.

Una vez finalizado el curso, se planteó analizar el uso de estas herramientas, considerando las ventajas e inconvenientes de las mismas mediante una encuesta que recabara la opinión de los estudiantes.

\subsection{Objetivos}

El objetivo principal de este estudio es analizar la adaptación de la docencia a un formato virtual provocada por la COVID-19, valorando la experiencia desde el punto de vista del alumnado a fin de detectar las prácticas y herramientas que han resultado de mayor utilidad. Nos gustaría matizar que siempre que hablemos de satisfacción del alumnado, nos referimos a una valoración global bajo su experiencia en el periodo analizado, y no 
sobre cuestiones específicas. Más concretamente, consideraremos los siguientes aspectos, a los cuales trataremos de dar respuesta:

- En primer lugar, nos interesa estudiar la satisfacción del alumnado con la adaptación de la docencia virtual a grandes rasgos. En concreto, cómo valoran de forma global la adaptación llevada a cabo por el profesorado, y qué tipo de docencia (presencial o virtual) prefieren recibir. Para esto nos planteamos dos preguntas específicas: ¿cómo valoran los estudiantes la docencia virtual? y ¿cuál es el grado de preferencia de la docencia virtual frente a la presencial?

- Nos interesa conocer qué tipo de docencia (síncrona o asíncrona) han recibido los alumnos, y cuál es su opinión respecto a estas dos estrategias en cuanto a cuál es más efectiva para su aprendizaje. Por tanto, realizamos las siguientes preguntas: ¿qué tipo de docencia han recibido mayoritariamente? y ¿cómo valoran la efectividad de ambas metodologías?

- Analizaremos también si la opinión del estudiante respecto a la docencia virtual y las diferentes estrategias y métodos utilizados varían significativamente en función de las distintas macroáreas del conocimiento. El objetivo es conocer si hay una diferencia significativa entre las opiniones de los estudiantes en función de la macroárea del conocimiento a la que pertenece el grado que están cursando.

- Por último, nos centraremos en una de las herramientas más empleadas para el desarrollo de la docencia síncrona: las plataformas de videoconferencia. La premura con la que se tuvo que adaptar la metodología, habida cuenta de la nueva situación, condujo a una falta de coordinación desde la universidad entre los distintos centros y departamentos. En consecuencia, los estudiantes han debido usar multitud de plataformas durante el desarrollo de la docencia virtual. Tenemos también como objetivo analizar qué herramientas han sido las más utilizadas, y conocer la opinión del alumnado sobre estas plataformas. Nuevamente, nos interesa conocer una valoración global del alumnado sobre las plataformas, no sobre cuestiones específicas relativas a las mismas. Para ello, nos interesa conocer qué plataforma ha sido la más usada y cuál se considera más idónea para la docencia síncrona, identificando qué factores facilitan la interacción con el profesorado y la adquisición de las competencias correspondientes a las asignaturas.

\subsection{Población y métodos}

Para la realización del proyecto, se confeccionó una encuesta que fue enviada a los coordinadores de los distintos grados de la UCO para su distribución entre el alumnado, lo que hace difícil conocer tanto el número exacto de estudiantes a los que llegó, como la tasa real de respuesta. Lo que sí es posible analizar es qué porcentaje de alumnos respecto a la matrícula del curso 2019/2020 respondió dicha encuesta, así como los grados y facultades/escuelas de pertenencia.

En total la encuesta ha sido respondida por 883 estudiantes de 34 grados distintos repartidos en 10 facultades y escuelas, lo que supone el 5,8\% del alumnado matriculado en dichas titulaciones según los datos publicados en el portal de la UCO (Datos de estudiantes matriculados, n. d.). 
Es importante matizar que a pesar de que el porcentaje mayor de los participantes se corresponde con las titulaciones de Ciencias Sociales y Jurídicas, estas son las que comprenden mayor volumen de alumnado, en concreto el $43 \%$ del alumnado de la UCO. De hecho, en términos relativos el mayor porcentaje de encuestados se encuentra en los grados de la macroárea Ingeniería y Arquitectura, puesto que esta solamente aglutina al $15,7 \%$ del estudiantado de la UCO.

\subsection{Comparativa de herramientas de videoconferencia}

Existe actualmente una enorme oferta de aplicaciones de videoconferencia que han estado a disposición del alumnado y profesorado. Antes de detallar y analizar las opiniones recabadas en la encuesta, nos interesa analizar, en particular, la versatilidad de las distintas herramientas, prestando especial atención a las plataformas en las que están disponibles, si son gratuitas o tienen limitaciones, así como su funcionalidad, valorando su facilidad de instalación y uso. Nos centramos en aquellas plataformas que los estudiantes han manifestado utilizar: Zoom, Cisco Webex, Blackboard Collaborate, Microsoft Teams, Google Meet/Hangouts, Jitsi Meet y Skype. Exponemos de cada una algunas ventajas y desventajas seleccionadas por su utilidad para la docencia. Al final de la sección, y a modo de resumen, se ofrece una tabla comparativa de estas.

\subsubsection{Zoom}

Zoom es una de las herramientas que, antes de la situación acaecida por la COVID-19, era una gran desconocida. Se popularizó mucho durante el mes de marzo, pero durante el mes de abril se publicaron diversas noticias que alertaron sobre problemas de privacidad y seguridad (CNN-CERT, 2020; García, 2020; Wakefield, 2020). Esto hizo que muchos usuarios se cuestionaran su uso. No obstante, a día de hoy y según la compañía responsable de Zoom, los problemas han sido solucionados y se están implementando mejoras en la seguridad (Linares, 2020).

- Pros: compatible con todos los sistemas operativos, facilidad de uso, herramientas útiles para docencia (pizarra, escribir en la pantalla, etc.), vista en galería de hasta 49 participantes a la vez. Permite hasta 100 usuarios conectados.

- Contras: llamadas gratuitas limitadas a 40 minutos, reiterados problemas de seguridad y privacidad.

\subsubsection{Cisco Webex}

Esta plataforma cuenta con la reputación de Cisco, por lo que uno de sus principales atractivos es su seguridad. Además, es una de las plataformas para las que la UCO da soporte de forma oficial.

- Pros: aplicación muy segura y con soporte oficial en la UCO. Hasta 100 usuarios en versión gratuita y vista en galería de hasta 25 .

- Contras: versión gratuita limitadas a 50 minutos, no dispone de aplicación para Linux. Pocas utilidades adaptadas a la docencia.

\subsubsection{Blackboard Collaborate}

Esta es otra de las alternativas con soporte oficial desde la UCO (Muñoz-Cecilia, n. d.). Esta aplicación está integrada en la plataforma Moodle por lo que los docentes pueden utilizarla directamente. Hay que destacar que para acceder a una sesión como invitado no es necesaria ninguna instalación previa, puesto que se accede directamente desde cualquier navegador web. 
- Pros: uso desde un navegador y se integra bien con Moodle, con soporte oficial en la UCO, diversas utilidades integradas para la docencia y un límite de hasta 250 usuarios por sesión.

- Contras: poco intuitiva, no es fácil de instalar (si no está disponible en el servidor de la universidad) y la vista en galería solo es posible en sesiones de hasta 4 participantes.

\subsubsection{Microsoft Teams}

También se encuentra entre las alternativas ofrecidas oficialmente desde la UCO. No es solo una aplicación para videoconferencia sino una plataforma unificada de comunicación y colaboración en la nube (ver por ejemplo González, 2018). La opción gratuita está muy limitada, pues no permite la opción de videoconferencia. Es por tanto necesario disponer de una suscripción para utilizar las opciones más interesantes para la docencia.

- Pros: herramienta colaborativa especialmente relevante en entornos empresariales, con soporte oficial en la UCO. Herramientas útiles adicionales para la docencia. Disponible en todos los sistemas operativos.

- Contras: no es gratuita y está bastante orientada a aplicaciones de Microsoft. Vista en galería bastante pobre.

\subsubsection{Google Meet/Hangouts}

Google dispone de dos herramientas bastante similares. A grandes rasgos, Google Meet es la herramienta integrada dentro de G Suite (conjunto de herramientas orientadas a empresa previa suscripción) mientras que Hangouts es la opción gratuita para el público general. De hecho, Google Meet es lo que antes de abril de 2020 se conocía como Hangouts Meet. Actualmente, hasta septiembre de 2020, las características premium orientadas a empresa son gratuitas. Posteriormente se podrá contar con una versión gratuita más limitada. Se puede consultar una visión detallada de ambas opciones en Google (2020).

- Pros: fácil de utilizar sin necesidad de instalar nada, vista en galería de hasta 16 participantes.

- Contras: no dispone de herramientas adicionales para la docencia. Limitaciones para la versión gratuita.

\subsubsection{Jitsi Meet}

Se trata de una herramienta libre y gratuita. Se puede usar desde un servidor propio o a través de salas disponibles en otras instituciones. Se usa desde un navegador web, sin embargo, desde Linux parece dar problemas. Por lo tanto, es una herramienta muy prometedora a la que le falta aún recorrido. Una descripción más completa puede consultarse en Iglesias (2020).

- Pros: herramienta de código libre y gratuita. Edición colaborativa y hasta 25 participantes en vista en galería.

- Contras: le falta aún bastante desarrollo. No tiene herramientas de anotación (pizarra). 


\subsubsection{Skype}

Skype de Microsoft es una de las veteranas de las aplicaciones para videollamada. Se trata de una aplicación de telecomunicaciones que funciona para chats de video, llamadas y mensajería instantánea. Permite compartir pantalla, aunque no es posible hacer anotaciones o disponer de una pizarra online, por lo que para docencia puede estar algo limitada en ese aspecto. No obstante, esta es una herramienta que también tiene su uso en entornos educativos mediante la herramienta Skype in the Classroom.

- Pros: está bastante desarrollada y depurada, de uso gratuito, aunque con posibilidades de extender funcionalidad mediante pago.

- Contras: la limitación a 50 participantes puede ser una restricción importante para la docencia en grupos muy numerosos. No tiene herramientas de anotación o utilidades adicionales.

En la tabla 1 se muestra a modo resumen las principales características de las plataformas de videoconferencia: disponibilidad en todos los sistemas operativos (S.O.), posibilidad de uso desde un navegador web, limitación de la duración de llamadas para opciones gratuitas, número máximo de participantes (versión gratuita / versión de pago), máximo número de participantes visibles en vista mosaico o galería y disponibilidad de herramientas adicionales para la docencia.

Tabla 1.

Resumen de las principales características de las plataformas de videoconferencia. (V) denota que verifica la característica con ciertas restricciones. Las aplicaciones marcadas con un asterisco son aquellas con soporte oficial desde la UCO.

\begin{tabular}{|c|c|c|c|c|c|c|}
\hline Plataforma & s.o. & Web & $\begin{array}{l}\text { Límite } \\
\text { Ilamadas }\end{array}$ & $\begin{array}{c}\text { Máx. } \\
\text { Participantes }\end{array}$ & $\begin{array}{c}\text { Vista } \\
\text { galería }\end{array}$ & $\begin{array}{c}\text { Herramientas } \\
\text { adicionales }\end{array}$ \\
\hline Zoom & V & & $40 \min$ & 100 & 49 & V \\
\hline Cisco Webex* & & $\mathrm{V}$ & $50 \mathrm{~min}$ & $100 / 200$ & 25 & $(\mathrm{~V})$ \\
\hline $\begin{array}{l}\text { Blackboard } \\
\text { Collaborate* }\end{array}$ & & V & & 250 & 4 & V \\
\hline $\begin{array}{l}\text { Microsoft } \\
\text { Teams* }\end{array}$ & V & V & & 250 & 9 & V \\
\hline $\begin{array}{l}\text { Google } \\
\text { Meet/Hangouts }\end{array}$ & V & V & $1 \mathrm{~h}$ & $100 / 250$ & 16 & \\
\hline Jitsi Meet & & $(\mathrm{V})$ & & 75 & 25 & \\
\hline Skype & $\mathrm{V}$ & V & $4 \mathrm{~h}$ & 50 & 10 & \\
\hline
\end{tabular}

Fuente: Elaboración propia. 


\section{RESULTADOS}

Para conocer la opinión de los alumnos sobre la virtualización de la docencia, se les solicito que rellenaran una encuesta online. Dicha encuesta se realizó mediante un formulario de Google (Google Forms), incluyendo 16 cuestiones de las cuales: 9 preguntas recababan información tanto del perfil del alumnado como de las herramientas utilizadas, y 7 preguntas buscaban determinar qué tipo de docencia virtual habían recibido, qué herramientas habían utilizado de forma más frecuente y su grado de satisfacción. Para analizar la frecuencia y el grado de satisfacción se utilizaron cuestiones con distintas escalas de tipo Likert de 5 puntos (véase el Anexo 1 para el listado de preguntas).

Dicha encuesta fue creada para dar respuesta a las cuestiones planteadas en la subsección 2.1 y que constituyen los objetivos del estudio, por lo que debe ser evaluada para ser validada. En las siguientes secciones analizaremos estas preguntas de forma independiente.

\subsection{Perfil del estudiante}

Para contextualizar nuestro estudio comenzamos analizando el perfil del alumnado encuestado: la titulación que estudian, años de experiencia en la universidad, calidad de su conexión a Internet, su disponibilidad de acceso a equipamiento informático a fin de poder desarrollar la docencia, etc.

En la figura 1 se muestran los 7 grados con mayor participación en la encuesta, y la figura 2 muestra la representación en nuestra encuesta de los distintos centros (escuelas y facultades) y macroáreas de conocimiento.

En concreto, podemos observar que hemos obtenido una participación representativa del alumnado distribuido según las cinco macroáreas de conocimiento oficialmente reconocidas: Artes y Humanidades, Ciencias, Ciencias de la Salud, Ciencias Sociales y Jurídicas e Ingeniería y Arquitectura. También es destacable que la encuesta realizada ha tenido mayor calado entre el alumnado con menos años en la universidad, como se puede observar en la figura 3.

A pesar de las desviaciones en términos relativos frente al número real de estudiantes matriculados en cada titulación y macroárea, ya comentadas en la subsección 2.2, consideramos que la muestra obtenida es adecuada y representativa. Por ello podemos suponer que las conclusiones obtenidas representan la opinión general del alumnado de la UCO. 


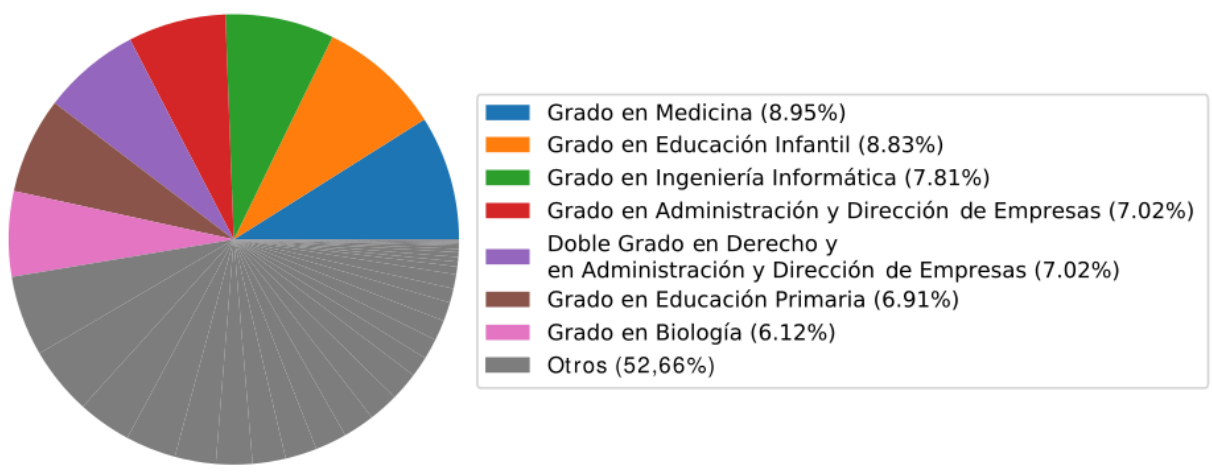

Figura 1. Titulaciones más representadas en la encuesta realizada. Otros se corresponde con el resto de las 27 titulaciones cuyos estudiantes han respondido la encuesta. Fuente: elaboración propia.
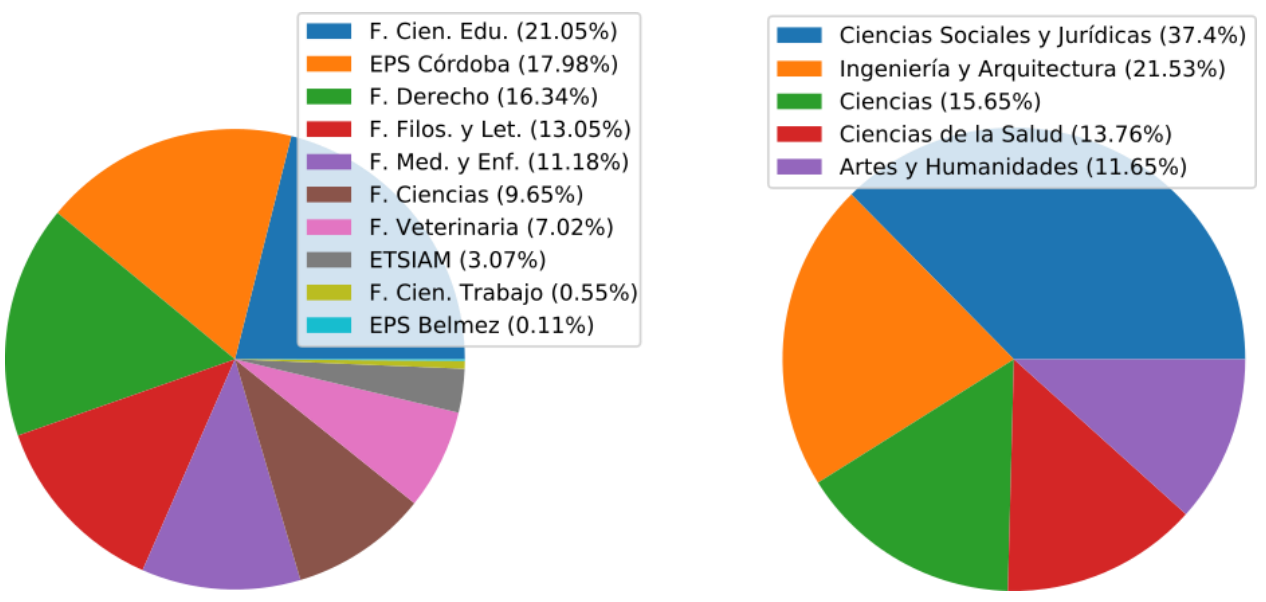

Figura 2. Representación de los centros (izquierda) y macroáreas de conocimiento (derecha) en la encuesta realizada. EPS y ETSIAM denotan Escuela Politécnica Superior y Escuela Técnica Superior de Ingeniería Agronómica y Montes, respectivamente. Fuente: elaboración propia.

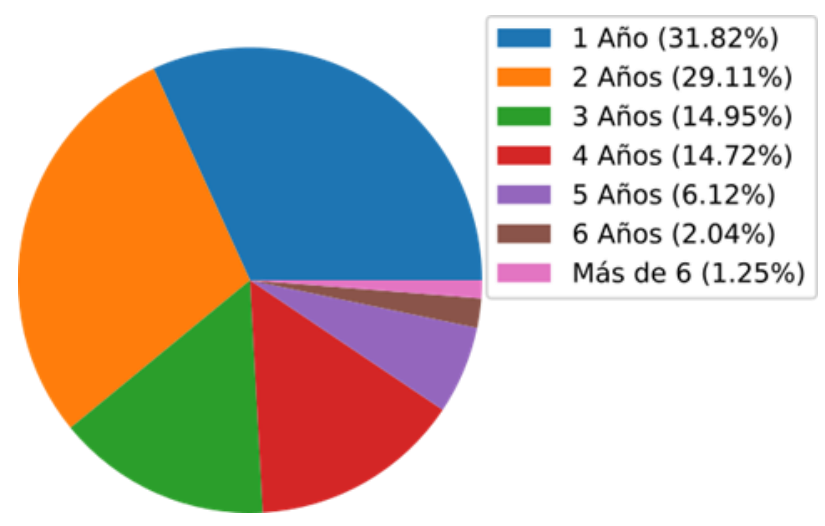

Figura 3. Años que llevan los encuestados estudiando en la UCO, de forma porcentual. Fuente: elaboración propia.

En lo referente a los medios técnicos que disponían los estudiantes, podemos observar en la figura 4 que tenían a su disposición, en general, una conexión de fibra, 
la cual consideraban (en una amplia mayoría) entre buena y muy buena. Por otro lado, nos parece interesante destacar que, según las respuestas obtenidas, una gran mayoría de los encuestados disponían de ordenador portátil (en concreto 815, lo que supone un $92.3 \%$ del total de los encuestados). También se ha comprobado que aquellos que no disponen del mismo, han contado con un ordenador de sobremesa. Todo esto, unido a que un $57 ., 3 \%$ de los encuestados manifiesta que dicho material es para su uso de forma exclusiva (es decir, no comparten este material), indica que la falta de medios materiales no parece un problema general entre los encuestados. Cabe destacar que al inicio del estado de alarma la UCO dotó a los estudiantes que lo solicitaron de equipos portátiles y tarjetas de datos en concepto de préstamo, por lo que no es de extrañar los datos extraídos de la encuesta.
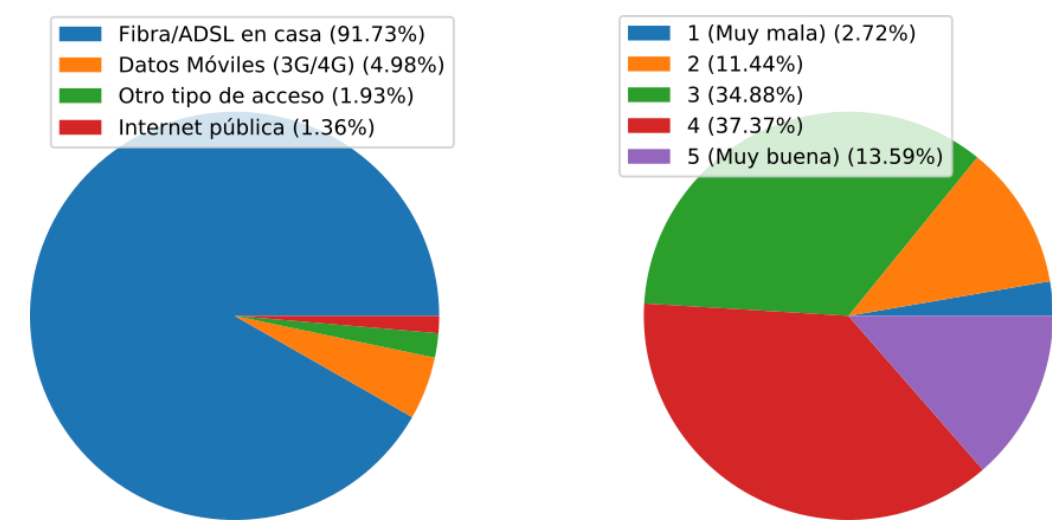

Figura 4. Tipo de conexión (izquierda), así como su calidad (derecha), usados por los estudiantes encuestados.

Fuente: elaboración propia.

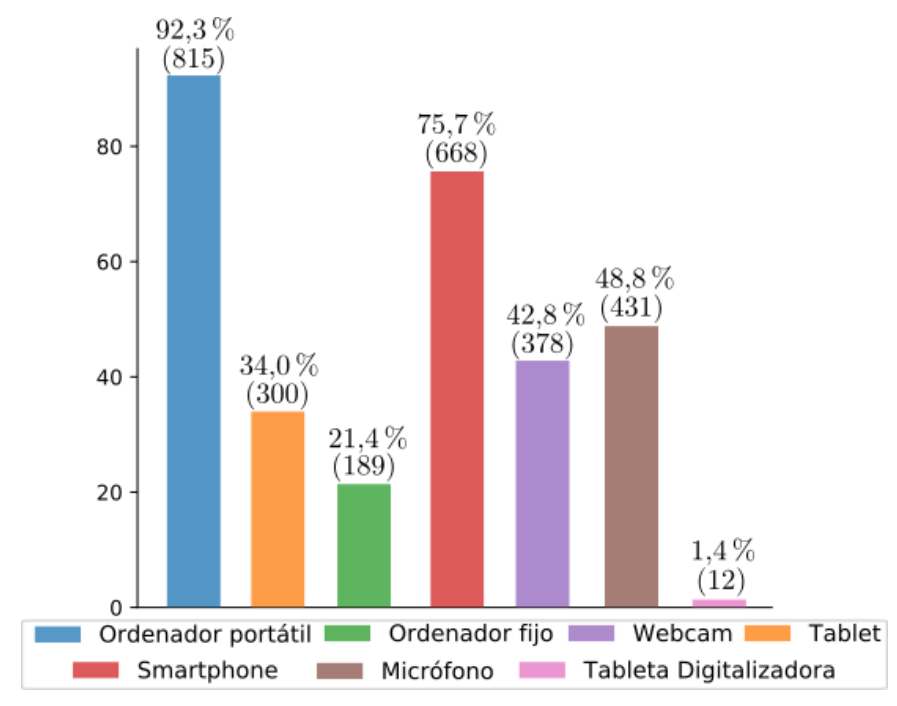

Figura 5. Número de estudiantes que disponen de los distintos materiales de trabajo, de forma no exclusiva.

Fuente: elaboración propia. 


\subsection{Satisfacción del alumnado respecto a la virtualización de la docencia}

Abordamos ahora la más general de nuestras preguntas, referente a la satisfacción global del alumnado respecto de la virtualización de la docencia. Con este fin, destacamos tres preguntas de la encuesta respondida por los estudiantes: ¿Cuál ha sido en general tu grado de satisfacción con la docencia online?, ¿Cómo ha sido tu experiencia de docencia online en comparación a la docencia presencial? y Valora en general la adaptación de la docencia presencial a la modalidad online.

La figura 6 muestra las respuestas a estas preguntas. Se extrae de los resultados que la docencia virtual no ha sido en general satisfactoria para los estudiantes. De hecho, solo un $15.6 \%$ de los encuestados valora dicha docencia de forma positiva (valoración >3). Este dato, combinado con que el $70.2 \%$ de ellos manifiesta preferencia por la docencia de tipo presencial frente a la virtual, nos hace concluir que, de forma general, el grado de satisfacción con la docencia virtual ha sido bajo, así como que una amplia mayoría de los encuestados se inclinan por una mayor efectividad de la docencia de tipo presencial. De las respuestas a la tercera pregunta, se concluye que el alumnado no cree que la docencia se haya adaptado correctamente a un formato virtual (tan solo un $14,8 \%$ de respuestas positivas). Esto concuerda con los resultados de las preguntas previas y podría estar relacionado con la alta preferencia por una docencia de tipo presencial.
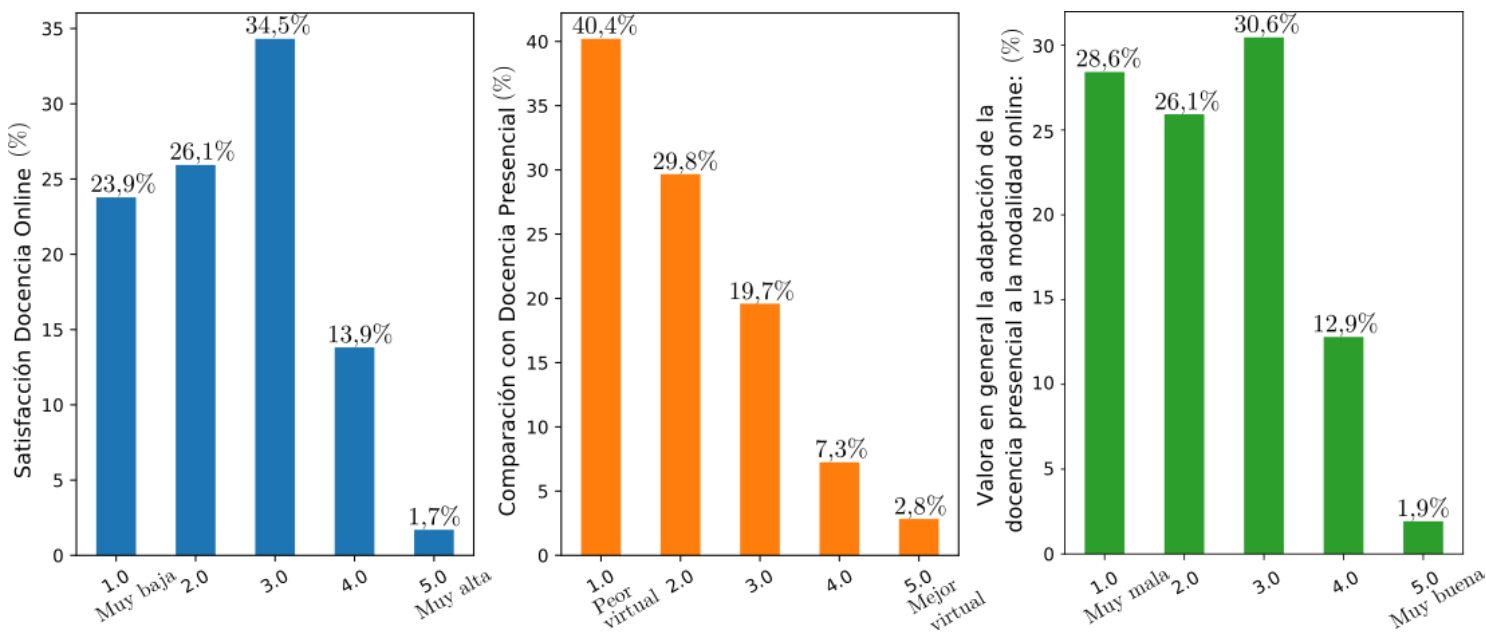

Figura 6. Izquierda: grado de satisfacción con la docencia virtual; centro: mismo grado de satisfacción cuando se compara con una docencia de tipo presencial; derecha: cómo ha sido la adaptación de la docencia a un formato virtual según los encuestados.

Fuente: elaboración propia.

\subsection{Preferencias del estudiante frente a docencia síncrona/asíncrona}

Ante la flexibilidad planteada por la UCO para impartir la docencia durante el cuatrimestre, el profesorado universitario ha podido optar por una docencia síncrona (clases por videoconferencia principalmente), asíncrona (apuntes, vídeos, presentaciones, ...), o una combinación de ambas. Para estudiar este aspecto, en la encuesta se han planteado dos preguntas: ¿Cómo clasificarías la docencia online que has recibido, principalmente síncrona, asíncrona, o ambos por igual? y ¿Cómo consideras que es más efectiva? Con la primera pregunta se pretende conocer el tipo de docencia más usado en este periodo, mientras que con la segunda se pretende conocer la opinión del alumnado sobre que opción es más eficaz para su aprendizaje. 
En la figura 7 mostramos las respuestas a las preguntas anteriores, donde vemos que solo un $13,7 \%$ de los encuestados han recibido principalmente docencia de tipo síncrona, mientras que un $52,2 \%$ de ellos se han beneficiado de un uso similar de ambos tipos de docencia. También vemos claro que la docencia asíncrona ha sido más usada por los docentes del alumnado encuestado, ya que un $86,3 \%$ de ellos afirma haber recibido dicha docencia en mayor o menor medida, frente a un 65,9\% que recibe algún tipo de docencia síncrona. Estos resultados contrastan con la opinión de los encuestados sobre cómo debería ser la docencia. Tan solo un 8,9\% de los encuestados consideran que una docencia principalmente asíncrona sería la mejor opción, a pesar de que ha sido lo recibido por un $34,1 \%$ de ellos. Un $69,1 \%$ de los estudiantes considera que se deberían combinar ambos tipos por igual, mientras que el 22,0\% considera que la docencia debería ser principalmente síncrona.
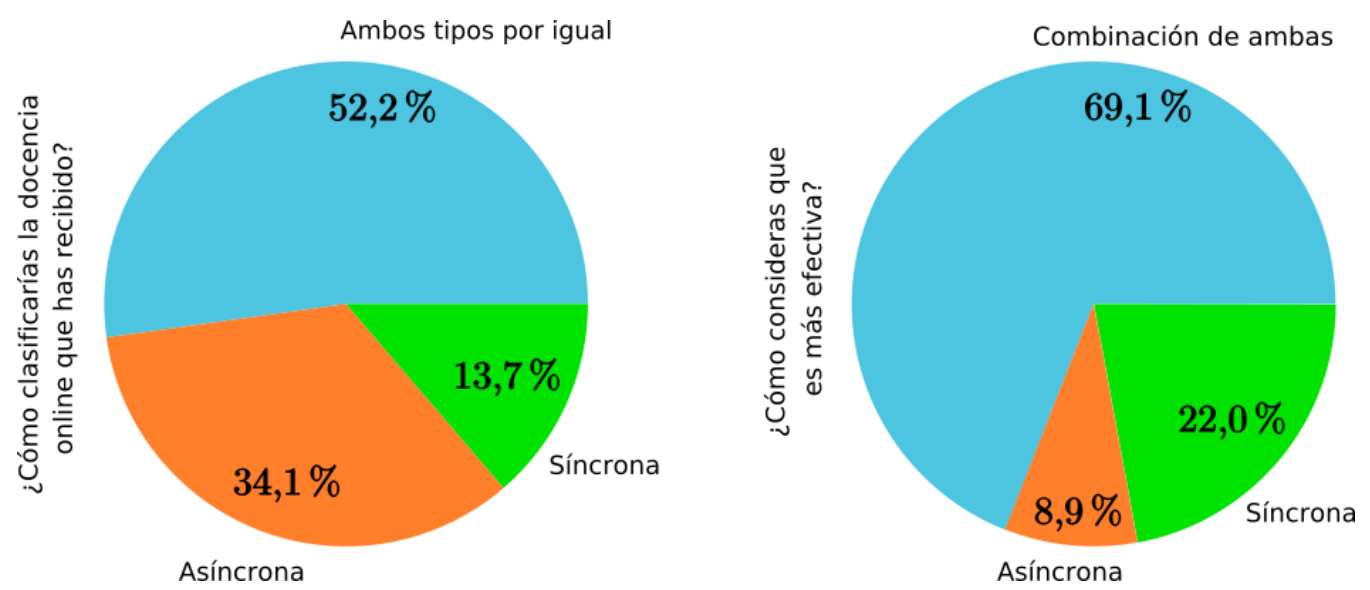

Figura 7. Clasificación de la docencia recibida por los encuestados, y cómo piensan que debería ser.

Fuente: elaboración propia.

\subsection{Variabilidad de los resultados obtenidos en función de las distintas macroáreas de conocimiento}

Nos preguntamos ahora si en las dos cuestiones anteriores ha habido diferencias dependiendo de la macroárea de conocimiento en la que se encuadra cada una de las titulaciones de los encuestados.

En las figuras 8 y 9 vemos las respuestas a todas las preguntas analizadas hasta el momento, en función de las macroáreas de conocimiento. La tendencia general es la mostrada en el análisis global que hemos realizado previamente: los estudiantes mayoritariamente no se muestran satisfechos con la docencia online, expresan su preferencia por una modalidad presencial, y consideran que la docencia no se ha adaptado correctamente al formato virtual. Sin embargo, vemos diferencias notables entre las macroáreas. 

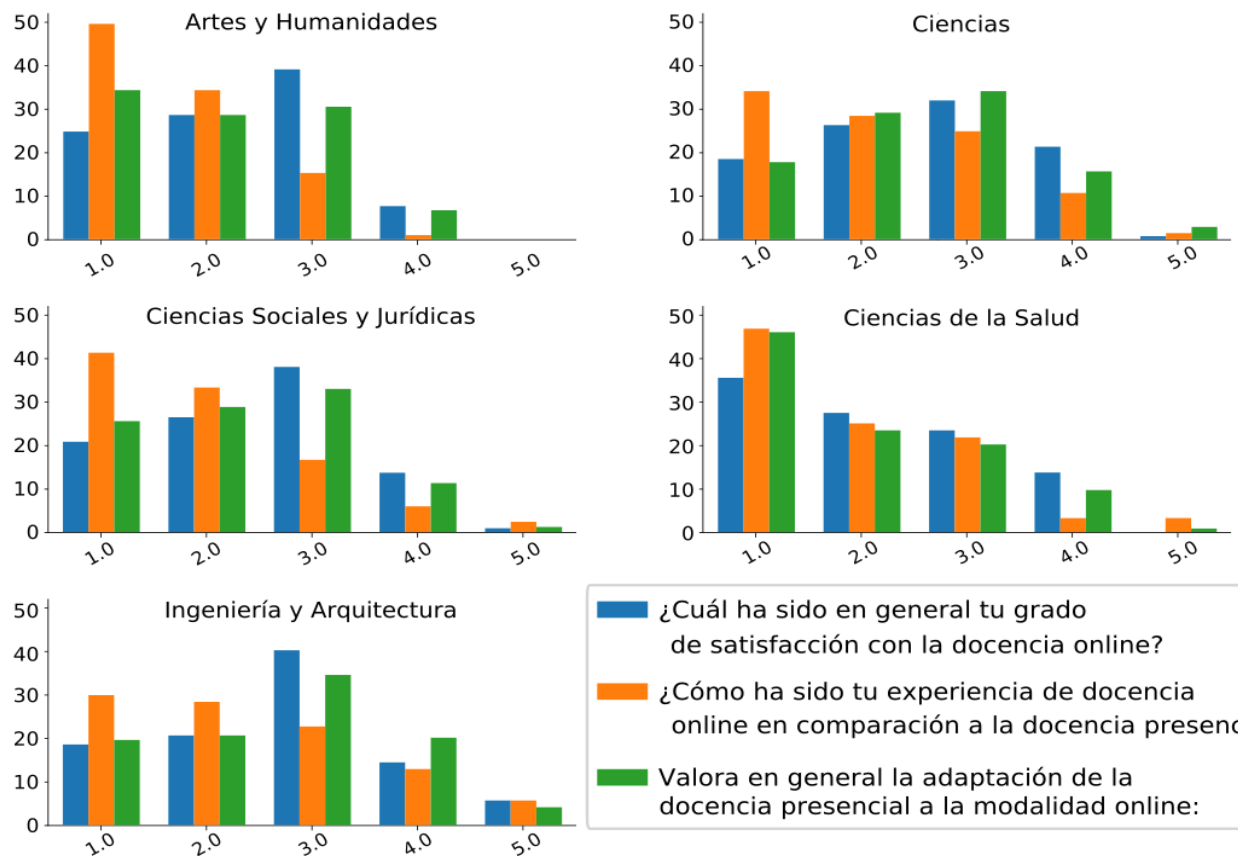

¿Cuál ha sido en general tu grado de satisfacción con la docencia online?

¿Cómo ha sido tu experiencia de docencia online en comparación a la docencia presencial?

Valora en general la adaptación de la docencia presencial a la modalidad online:

Figura 8. Satisfacción global (1-opción menos favorable, 5-opción más favorable) de forma porcentual con la docencia virtual en función del área de conocimiento.

Fuente: elaboración propia.

En la tabla 2 mostramos los resultados a las tres preguntas relativas a la satisfacción del alumnado con la docencia virtual, agrupando las respuestas como positivas o negativas, en función de si la valoración es mayor o menor que 3 . Mirando esta tabla, junto a la figura 8 , vemos de forma clara que los estudiantes de Ciencias e Ingeniería y Arquitectura son los que manifiestan una mayor satisfacción con la docencia online, un menor perjuicio de la docencia online frente a la presencial, y opinan que ha habido una mayor adaptación de la docencia presencial a la virtual, aun siendo estas valoraciones negativas. En el lado opuesto se encuentran las titulaciones de Ciencias de la Salud y en menor medida las de Artes y Humanidades, cuyos estudiantes muestran un mayor descontento tanto con la docencia online como con la adaptación a esta modalidad. En todas las áreas hay preferencia por la docencia presencial, siendo esta tendencia ligeramente más leve en Ingeniería y Arquitectura, posiblemente debido a que piensan que la docencia se ha adaptado mejor. 
Tabla 2.

Porcentaje de respuestas positivas (>3) y negativas (<3) a las preguntas relativas a la satisfacción respecto a la docencia virtual: ¿Cuál ha sido en general tu grado de satisfacción con la docencia online?, ¿Cómo ha sido tu experiencia de docencia online en comparación a la docencia presencial? y Valora en general la adaptación de la docencia presencial a la modalidad online. A.H. denota Artes y Humanidades, C. Ciencias, C. S. Ciencias de la Salud, C.S.J. Ciencias Sociales y Jurídicas, y I.A. Ingeniería y Arquitectura.

\begin{tabular}{lcccccc}
\hline \multicolumn{1}{c}{ Pregunta } & & A.H. & C. & C.S. & C.S.J. & I.A. \\
\hline Grado satisfacción & $(>3)$ & 7,6 & 22,3 & 13,7 & 14,6 & 20,2 \\
\hline Docencia Online vs Presencial & $(<3)$ & 53,3 & 45,3 & 62,9 & 47,3 & 39,4 \\
\hline & $(>3)$ & 1,0 & 12,1 & 6,5 & 8,4 & 18,7 \\
\hline Adaptación de la docencia & $(<3)$ & 83,8 & 62,9 & 71,8 & 74,9 & 58,5 \\
\hline & $(>3)$ & 6,7 & 18,6 & 10,5 & 12,5 & 24,5 \\
\hline
\end{tabular}

Fuente: Elaboración propia.

Respecto al tipo de docencia, observando la figura 9 vemos que los estudiantes opinan de forma unánime en todas las áreas que una combinación de docencia síncrona y asíncrona sería lo más efectivo para su aprendizaje, aunque los estudiantes de Artes y Humanidades valoran en mayor medida que el resto de áreas la docencia asíncrona. Esto contrasta con el tipo de docencia que manifiestan haber recibido. En general, se ha utilizado una combinación de ambos tipos, aunque en un porcentaje menor del que cabría esperar. Es llamativo el caso de las titulaciones de Ciencias de la Salud, donde un $67.7 \%$ de los estudiantes manifiestan haber recibido principalmente docencia asíncrona, y únicamente para un $4.0 \%$ de los encuestados la principal ha sido de tipo síncrona.

En general, podemos concluir que, dentro del descontento general de los estudiantes, los de titulaciones de las áreas de Ciencias e Ingeniería y Arquitectura son los que manifiestan resultados más positivos, tanto en la adaptación de la docencia, como con la satisfacción general. 

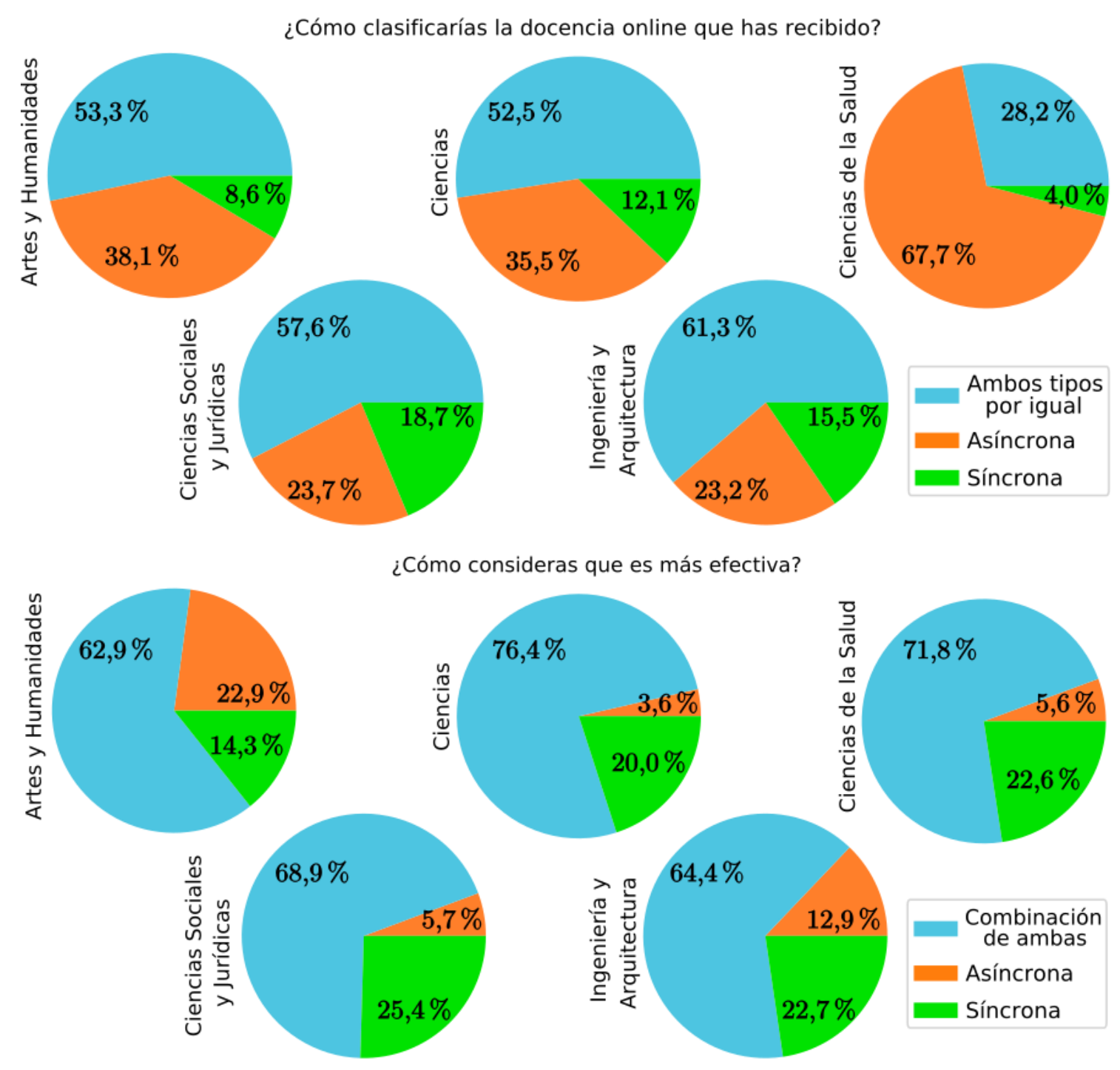

Figura 9. Clasificación de la docencia recibida por los encuestados, y cómo piensan que debería ser, dependiendo del área de conocimiento.

Fuente: elaboración propia.

\subsection{Valoración y uso de las distintas plataformas de comunicación}

Ante lo imprevisto de esta situación, ha sido necesario buscar las herramientas más adecuadas para poder adaptar la docencia, e impartirla de forma remota.

En lo que sigue tratamos de discutir cuales han sido las opciones escogidas por el profesorado de los encuestados, así como la valoración que los estudiantes hacen de dichas plataformas. Consideramos aquí las plataformas analizadas en la subsección 2.3. Añadimos también YouTube que, aunque no es una herramienta de videoconferencia, resulta de utilidad como herramienta de comunicación.

Para estudiar las cuestiones relacionas con estas plataformas, los estudiantes han respondido a las preguntas: ¿Con qué frecuencia has utilizado las siguientes plataformas y aplicaciones? y Valora las distintas plataformas y aplicaciones utilizadas.

Las figuras 10 y 11 muestran las respuestas de los encuestados sobre la frecuencia de uso de las distintas plataformas. Como puede observarse, un $85.2 \%$ de 
los encuestados manifiestan haber usado Zoom, lo que la convierte en la plataforma más utilizada, seguida por Blackboard Collaborate (72.7\%), y Cisco Webex (59.3\%). Para el resto de ellas, más de la mitad de los estudiantes indican que no las han usado, destacando por su escaso uso Google Meet/Hangouts y Jitsi Meet que han sido utilizadas por un $10.5 \%$ y $12.7 \%$ de los encuestados. Destacable también que Microsoft Teams ha sido usada por un $37.6 \%$ de los encuestados, a pesar de ser una de las tres herramientas recomendadas, y con soporte, por la UCO. Este orden de plataformas más usadas no se altera si se considera solo aquellos estudiantes que manifiestan un uso relativamente frecuente (puntuación mayor o igual a 3).

En lo anterior, únicamente hemos tenido en cuenta si un estudiante indica haber usado una plataforma, independientemente de la frecuencia. Si miramos este indicador, vemos que únicamente Zoom y Blackboard Collaborate tienen más estudiantes que indican haber usado mucho (usuarios habituales, suma de valores 4 y 5 en figura 11) dicha plataforma, que estudiantes que indican haberla usado poco (usuarios esporádicos, suma de valores 1 y 2 en figura 11). Para las demás, es mayor el número de usuarios esporádicos que habituales, lo cual resulta llamativo.

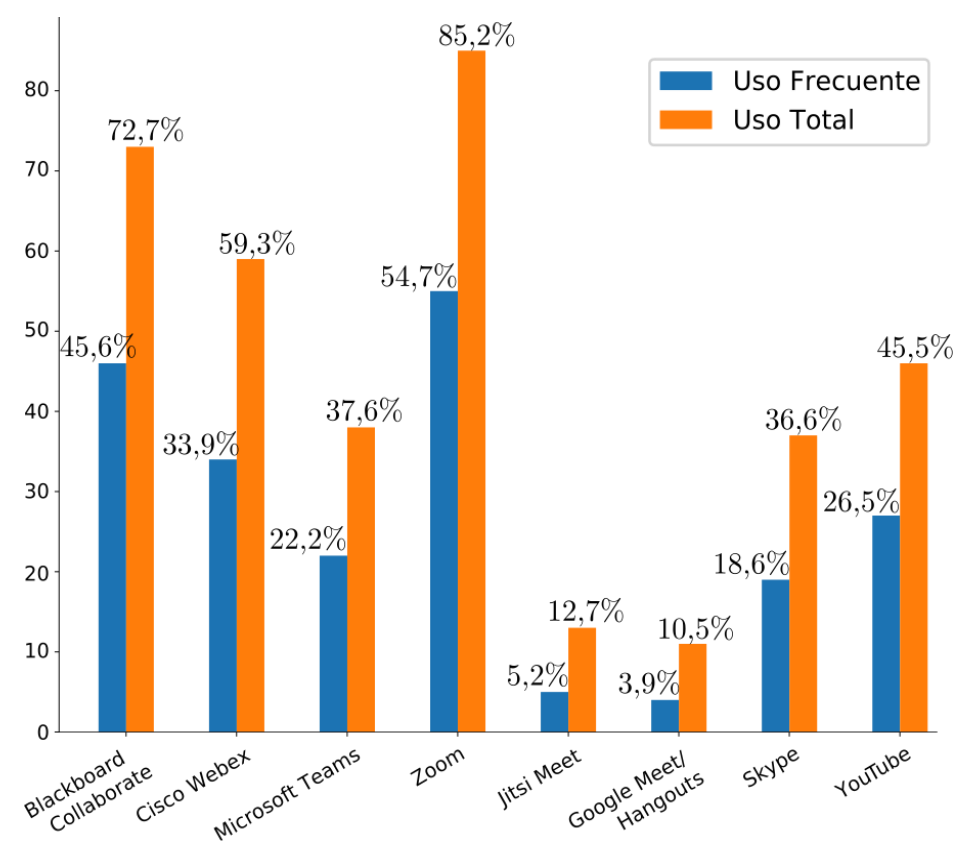

Figura 10. Frecuencias relativas de uso de las distintas plataformas según uso frecuente (puntuación mayor o igual a 3) y total.

Fuente: elaboración propia. 


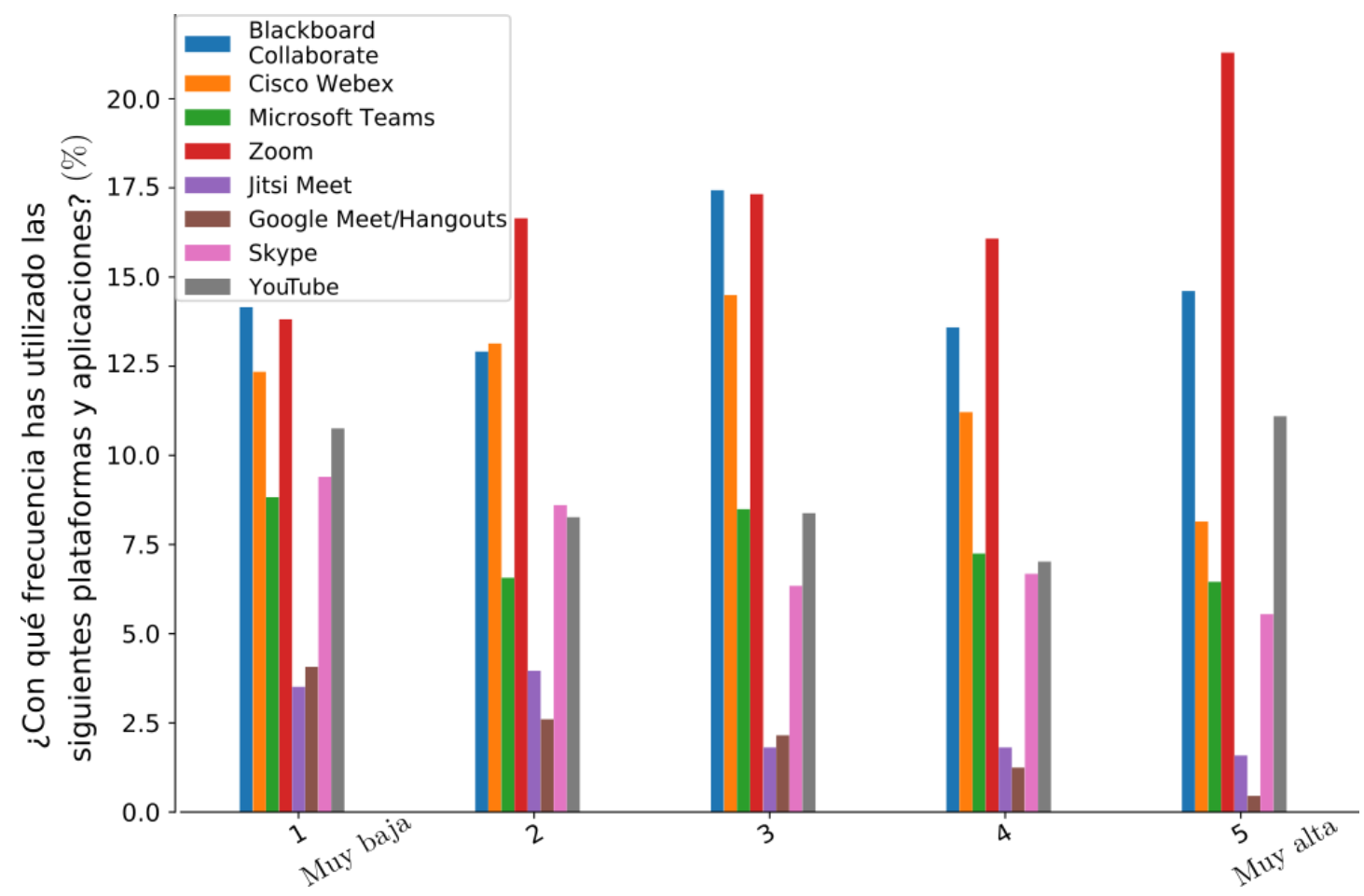

Figura 11. Frecuencias relativas de uso de las distintas plataformas.

Fuente: elaboración propia.

Una vez sabemos qué plataformas han sido las más utilizadas, veamos cómo son valoradas por los estudiantes. La figura 12 muestra el grado de satisfacción con las mismas, de forma porcentual respecto al número de encuestados que usa dicha plataforma. De forma general, para todas las plataformas, excepto Jitsi Meet, las valoraciones positivas (suma de valores 4 y 5 en figura 12) son mayores que las valoraciones negativas (suma de valores 1 y 2 en figura 12). En concreto en torno al $40 \%$ frente al $33 \%$. Destaca especialmente YouTube, para la cual las valoraciones positivas suman un $58,9 \%$, lo que la convierte en la plataforma mejor valorada, a pesar de no ser una herramienta de videoconferencia, si bien únicamente ha sido utilizada por un $45,5 \%$ (26,5\% de forma frecuente) de los encuestados.

A la vista de todos los datos sobre la frecuencia de uso y la valoración, parece claro que la plataforma que ha llevado el mayor peso de la docencia durante este periodo es Zoom, además con un grado alto de satisfacción por parte del alumnado. Indicar también que esto ha sucedido partiendo Zoom en desventaja por varias razones. En primer lugar, era una plataforma notablemente menos conocida que Microsoft (Teams o Skype), Cisco, o Google. En segundo lugar, no ha sido una aplicación con soporte desde la UCO, aunque durante las primeras semanas de confinamiento fue una de las aconsejadas. Además, como se indica en la sección 4 existían problemas de seguridad, que más tarde fueron solventados. 


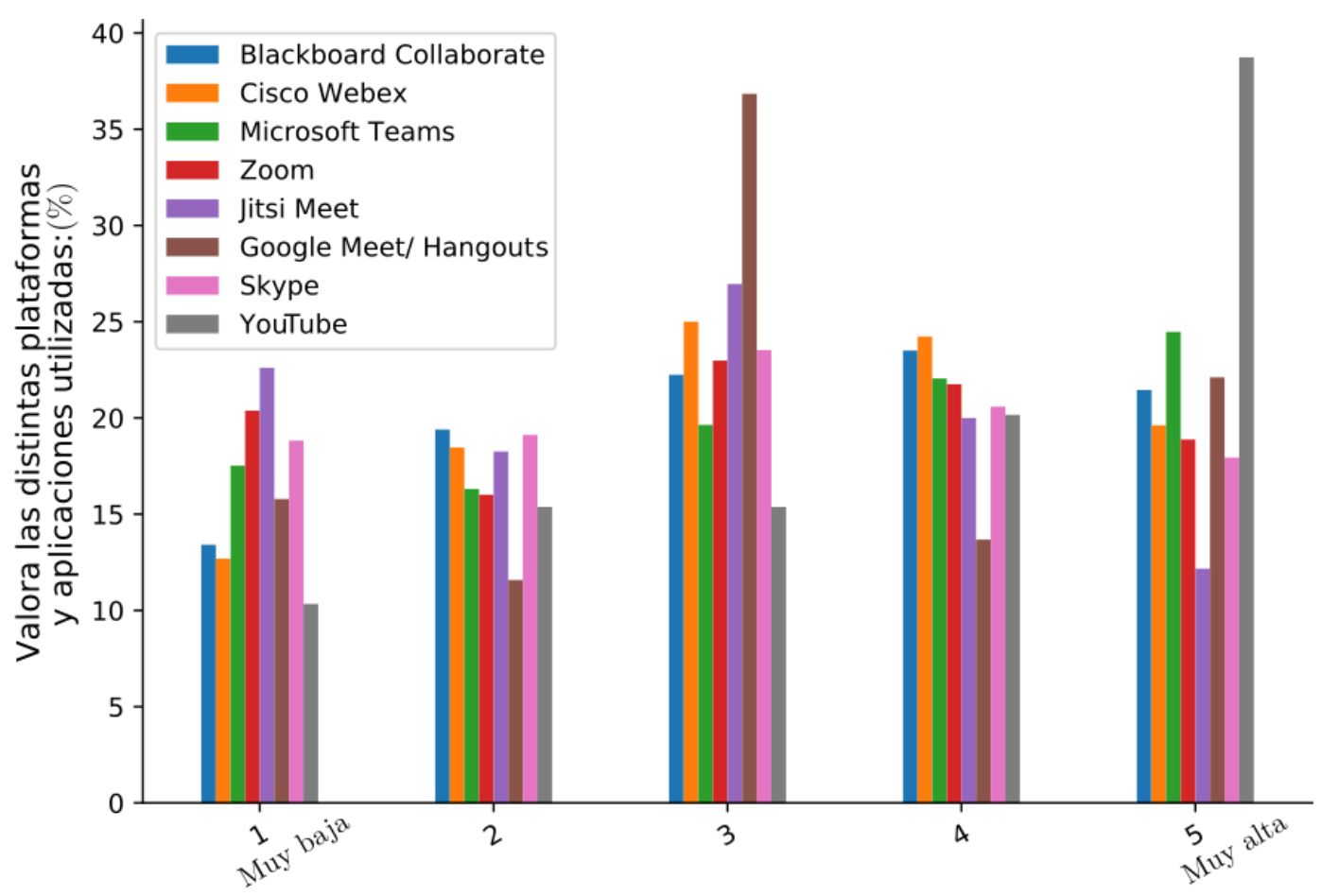

Figura 12. Valoraciones relativas de uso de las distintas plataformas. Cada porcentaje se calcula respecto al total de usuarios de cada una de las plataformas.

Fuente: elaboración propia.

\section{CONCLUSIONES}

En este estudio se analiza cómo ha sido la adaptación de la docencia a un formato online realizada por los docentes de la Universidad de Córdoba durante la crisis sanitaria de la COVID-19. Esta situación atípica se ha traducido en el uso de distintas y muy variadas estrategias para la virtualización de la docencia, lo cual nos permite obtener una visión muy general de la efectividad de las distintas aproximaciones realizadas, así como algunas conclusiones de interés para la organización futura de la docencia.

Lo primero a destacar ha sido la gran participación que la encuesta ha tenido, siendo respondida por 883 estudiantes de perfiles muy variados. Esto nos sugiere que los resultados de la misma pueden ser extensibles, no solo a todo el alumnado de la UCO, sino también a otras instituciones de educación superior. Entre los encuestados no hay problemas destacables respecto al material, disponiendo todos de equipamiento (ya sea ordenador de sobremesa, portátil o tablet). Remarcar que un $92.3 \%$ de los encuestados manifiesta disponer de ordenador portátil, y un $57 \%$ disfruta dispone del material de forma exclusiva (no lo comparte). Por otro lado, solo un $14.2 \%$ del alumnado declara tener un tipo de conexión que podría considerarse insuficiente para una docencia virtual completa.

Desde un punto de vista general podemos concluir que el alumnado muestra, en mayor o menor medida, descontento con la virtualización de la docencia que se ha llevado a cabo en la UCO. Una amplia mayoría de los encuestados consideran que la docencia no ha sido adaptada convenientemente, valorándola negativamente, y 
mostrando preferencia por la docencia presencial. Esta valoración negativa es menor en macroáreas más técnicas, como son Ciencias e Ingeniería y Arquitectura, donde la percepción general es que los alumnos suelen tener una mayor inclinación hacia las nuevas tecnologías.

Con respecto a la virtualización de la docencia, es destacable que ha existido una cierta inclinación del profesorado de la UCO por la docencia asíncrona por encima de la síncrona. Destaca el caso de las titulaciones de Ciencias de la Salud, donde la docencia de tipo asíncrona ha sido ampliamente más utilizada como tipo principal de docencia. Esto puede deberse al régimen especial de una parte del profesorado que imparte docencia en estas titulaciones.

Sin embargo, los datos muestran que el alumnado de todas las macroáreas solicita una aproximación híbrida, donde la docencia síncrona también tenga un peso importante, aunque complementada adecuadamente con otros recursos de tipo asíncrono. Se extrae por tanto que toda virtualización efectiva de la docencia debe tener un componente importante de docencia por videoconferencia.

Respecto a las herramientas de virtualización más utilizadas, destacaríamos Zoom $(85.2 \%$ de uso global) y YouTube. La primera era una herramienta de videoconferencia poco conocida antes de la emergencia sanitaria y cuyo uso explotó al principio de la pandemia. Solamente Blackboard Collaborate ( $72.7 \%$ de uso global), la cual tiene soporte oficial desde la UCO y está integrada en el propio Moodle de la UCO, tiene un uso cercano al de Zoom.

En definitiva, queda patente la necesidad de promover acciones que mejoren la adaptación de la docencia a un entorno virtual. Parece indispensable que esta docencia incluya tanto herramientas para la docencia asíncrona (creación de notas y presentaciones, vídeos, etc.) como sesiones síncronas (uso de herramientas de videoconferencia principalmente), para lograr una mayor efectividad. 


\section{ANEXO 1. ENCUESTA REALIZADA A LOS ESTUDIANTES}

Con el fin de complementar el presente estudio, incluimos aquí la encuesta realizada a los estudiantes para la elaboración del mismo.

Dicha encuesta consta de 9 preguntas para la elaboración del perfil de los estudiantes, incluyendo el material disponible por los mismos:

\section{Perfil del Alumno}

Describimos distintos parámetros de interés sobre el alumno

Titulación *

Sélectionner

Edad: *

Votre réponse

¿Cuántos años llevas estudiando en la Universidad (incluyendo el actual)? *

Sélectionner

Figura 13. Preguntas 1-3 de la encuesta. Fuente: elaboración propia. 
Género: *

Hombre

Mujer

Prefiero no decirlo

Tipo de acceso a Internet que utilizas: *

Fibra/ADSL en casa

Datos Móviles (3G/4G)

Internet pública

Otro tipo de acceso

¿Cómo calificarias tu conexión a Internet? *

$\begin{array}{ccccccc}1 & 2 & 3 & 4 & 5 & \\ \text { Mala } & \bigcirc & \bigcirc & \bigcirc & \bigcirc & \bigcirc & \text { Excelente }\end{array}$

Figura 14. Preguntas 4-6 de la encuesta. Fuente: elaboración propia. 
¿De qué material dispones? *

$\square$ Ordenador fijo

$\square$ Ordenador portatil

$\square$ Tablet

$\square$ Smartphone

$\square$ Webcam

$\square$ Micrófono

$\square$ Tableta Digitalizadora

¿Tienes que compartir el material anterior con otras personas? *

Sí

$\bigcirc$ No

¿Habias tenido experiencia previa con cursos online?*

Sí

No

Figura 15. Preguntas 7-9 de la encuesta. Fuente: elaboración propia. 
A continuación, 7 preguntas para determinar el tipo docencia virtual habían recibido, así como las herramientas habían utilizado de forma más frecuente y su grado de satisfacción con la adaptación de la docencia a un formato online:

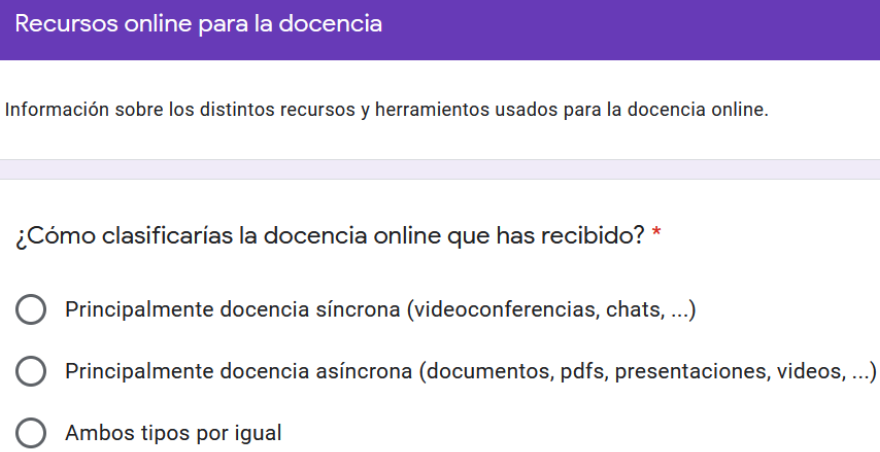

\begin{tabular}{|c|c|c|c|c|c|c|}
\hline & No usada & 1 & 2 & 3 & 4 & 5 \\
\hline $\begin{array}{l}\text { Blackboard } \\
\text { Collaborate }\end{array}$ & $\bigcirc$ & $\bigcirc$ & 0 & 0 & 0 & 0 \\
\hline Cisco Webex & 0 & 0 & $\bigcirc$ & 0 & 0 & O \\
\hline $\begin{array}{l}\text { Microsoft } \\
\text { Teams }\end{array}$ & $\bigcirc$ & 0 & $\bigcirc$ & $\bigcirc$ & $\bigcirc$ & $\bigcirc$ \\
\hline Zoom & $\bigcirc$ & 0 & 0 & $\bigcirc$ & $\bigcirc$ & $\bigcirc$ \\
\hline Jitsi Meet & 0 & 0 & 0 & 0 & 0 & 0 \\
\hline $\begin{array}{l}\text { Google Meet/ } \\
\text { Hangout }\end{array}$ & $\bigcirc$ & 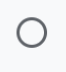 & $\bigcirc$ & $\bigcirc$ & $\bigcirc$ & $\bigcirc$ \\
\hline Skype & $\bigcirc$ & $\bigcirc$ & $\bigcirc$ & $\bigcirc$ & $\bigcirc$ & $\mathrm{O}$ \\
\hline Youtube & $\bigcirc$ & $\bigcirc$ & $\bigcirc$ & $\bigcirc$ & $\bigcirc$ & O \\
\hline EdPuzzle & $\bigcirc$ & $\bigcirc$ & $\bigcirc$ & $\bigcirc$ & $\bigcirc$ & $\bigcirc$ \\
\hline
\end{tabular}

Figura 16. Preguntas 10-11 de la encuesta. Fuente: elaboración propia. 
Valora las distintas plataformas y aplicaciones utilizadas: (1: mal, 5: excelente)

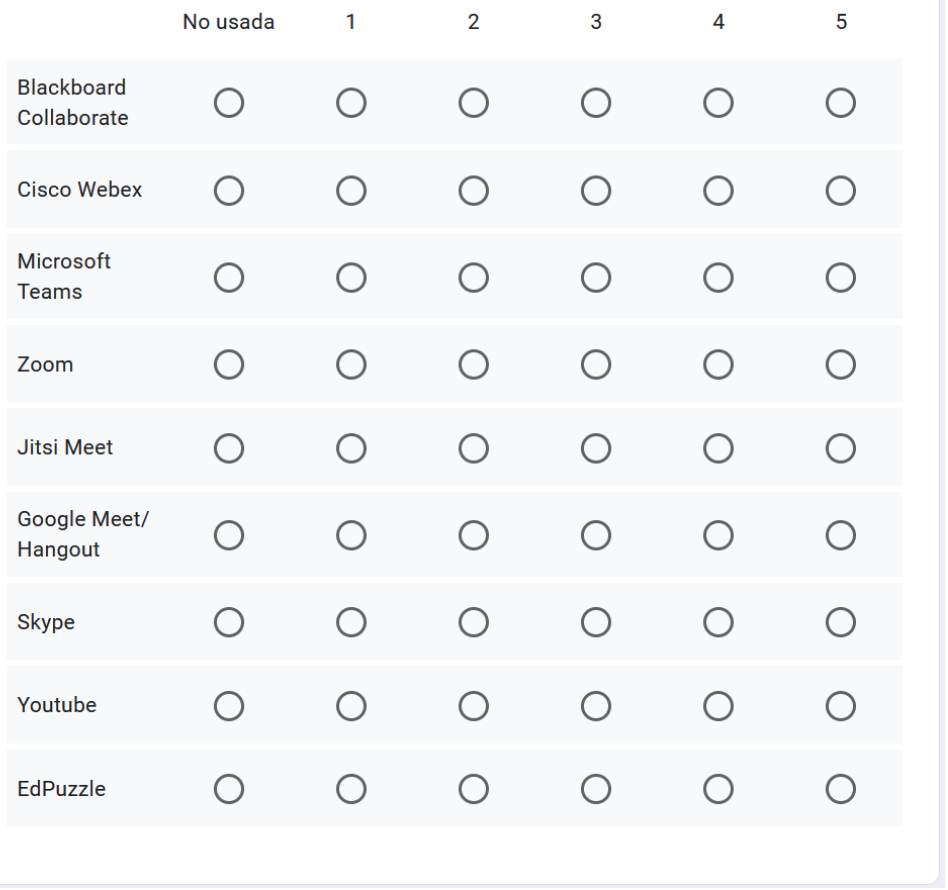

Figura 17. Preguntas 12 de la encuesta. Fuente: elaboración propia. 
Opinión sobre la docencia online

Valoraciones sobre la satisfacción con la docencia online y los distintos recursos empleados.

¿Cómo consideras que es más efectiva?

Síncrona (videoconferencias, chats, ...)

Asíncrona (documentos, pdfs, presentaciones, videos, ...)

Una combinación de ambas

¿Cuál ha sido en general tu grado de satisfacción con la docencia online?

$\begin{array}{lllllll} & 1 & 2 & 3 & 4 & 5 & \\ \text { Mala } & \bigcirc & \bigcirc & \bigcirc & \bigcirc & \bigcirc & \text { Excelente }\end{array}$

¿Cómo ha sido tu experiencia de docencia online en comparación a la docencia presencial?

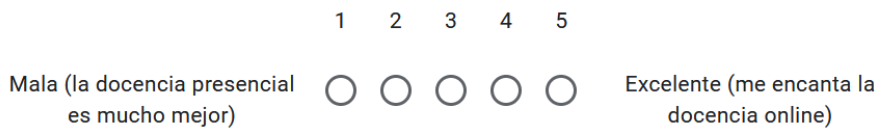

Valora en general la adaptación de la docencia presencial a la modalidad online:

$\begin{array}{ccccccc}1 & 2 & 3 & 4 & 5 & \\ \text { Mala } & \bigcirc & \bigcirc & \bigcirc & \bigcirc & \bigcirc & \text { Excelente }\end{array}$

Figura 18. Preguntas 13-16 de la encuesta. Fuente: elaboración propia.

\section{REFERENCIAS}

CNN-CERT. (2020). AV 22/20 Vulnerabilidad en Zoom. https://bit.ly/2Zyuazl.

Datos de estudiantes matriculados. (n. d.). Universidad de Córdoba. https://bit.ly/3umHkha.

García, J.G. (2020, 7 de abril). Los problemas de privacidad y seguridad sacuden el éxito de Zoom. El País. https://bit.ly/3sjbXm7.

Gisbert, M., \& Esteve, F. (2011). Digital learners: la competencia digital de los estudiantes universitarios. La Cuestión Universitaria, 7, 48-59.

González, J. (2018, 5 de agosto). Build a Collaborative Classroom with Microsoft Teams. Cult of Pedagogy. Recuperado de https://bit.ly/2Nu2idB.

Google (2020). Cambiar a Meet desde la versión clásica de Hangouts. https://bit.ly/3ql9QYn. 
Grupo de trabajo de directores TI. (2017). TIC 360: Transformación Digital en la Universidad. Madrid: CRUE Universidades Españolas. https://bit.ly/3scyzVc.

Iglesias, L.M. (2020, 23 de abril). Jitsi, videoconferencias educativas seguras a un clic. Cedec. https://bit.ly/2ZBBj2a.

Informe de situación de las tecnologías educativas en las Universidades Españolas 2018. (2018). En J. Gómez (Ed.), Madrid: CRUE Universidades Españolas. https://bit.ly/3kgApl9.

Linares, I. (2020, 18 de junio). Las videollamadas de Zoom estarán cifradas de extremo a extremo para todos los usuarios. Xataka móvil. https://bit.ly/3pK7dnz.

López-Pérez, M.V., Pérez-López, M.C., \& Rodríguez-Ariza, L. (2011). Blended learning in higher education: Students' perceptions and their relation to outcomes. Computers \& Education, 56 (3), 818-826.

Muñoz Cecilia, J. A. (n. d.). Blackboard Collaborate Ultra. UCOdigital: Centro de recursos virtuales. https://bit.ly/2NuYhp7.

Newman, T., Beetham, H. (2017). Student digital experience tracker 2017: the voice of 22,000 UK learners. Jisc. https://bit.ly/3dzl263.

The 2018 digital university: Staying relevant in the digital age. (n. d.). PwC. https://pwc.to/3aG57kl.

Universidad 2020: Papel de las TIC en el nuevo entorno socioeconómico. (2012). Fundación Telefónica. https://bit.ly/3bwY8d4.

UNIVERSITIC 2017. Análisis de las TIC en las Universidades Españolas. (2017). En J. Gómez (Ed.), Madrid: CRUE Universidades Españolas. https://bit.ly/3uk4vsE.

UNIVERSITIC 2016. Análisis de las TIC en las Universidades Españolas. (2016). En J. Gómez (Ed.), Madrid: CRUE Universidades Españolas. https://bit.ly/3dLCOmT.

Wakefield, J. (2020, 2 de abril). Zoom boss apologises for security issues and promises fixes. $B B C$ News. https://bbc.in/37A5cnU. 